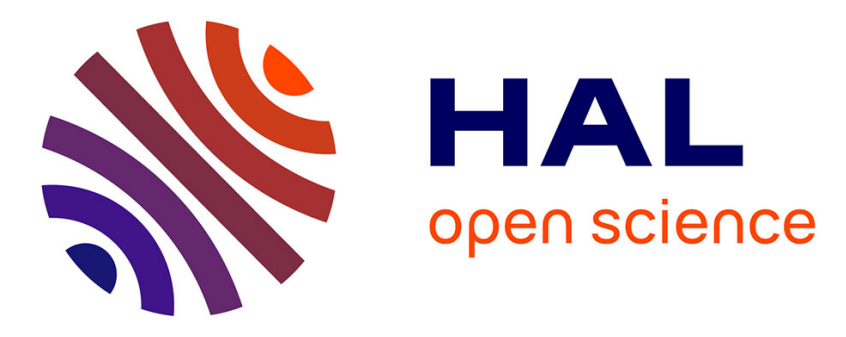

\title{
Enhancement of particle trapping in the Free Electron Laser
}

\author{
Romain Bachelard, Andrea Antoniazzi, Cristel Chandre, Duccio Fanelli, \\ Michel Vittot
}

\section{- To cite this version:}

Romain Bachelard, Andrea Antoniazzi, Cristel Chandre, Duccio Fanelli, Michel Vittot. Enhancement of particle trapping in the Free Electron Laser. Chaos06, Jun 2006, France. pp.73-77. hal-00102385

\section{HAL Id: hal-00102385 \\ https://hal.science/hal-00102385}

Submitted on 29 Sep 2006

HAL is a multi-disciplinary open access archive for the deposit and dissemination of scientific research documents, whether they are published or not. The documents may come from teaching and research institutions in France or abroad, or from public or private research centers.
L'archive ouverte pluridisciplinaire HAL, est destinée au dépôt et à la diffusion de documents scientifiques de niveau recherche, publiés ou non, émanant des établissements d'enseignement et de recherche français ou étrangers, des laboratoires publics ou privés. 


\title{
ENHANCEMENT OF PARTICLE TRAPPING IN THE FREE ELECTRON LASER
}

\author{
R. Bachelard *, A. Antoniazzi **, C. Chandre*, \\ D. Fanelli ${ }^{* *}$, M. Vittot* \\ * Centre de Physique Théorique, CNRS Luminy, Case 907, \\ F-13288 Marseille Cedex 9, France ${ }^{1}$ \\ ** Dipartimento di Energetica and CSDC, Università di \\ Firenze, INFN, via S. Marta, 3, 50139 Firenze, Italy
}

\begin{abstract}
The saturated dynamics of a Single Pass Free Electron Laser is here considered within a simplified mean field approach. A method is proposed to increase the size of the macro-particle. This approach is based on the reconstruction of invariant tori of the dynamics of test particles. To this aim a dedicated control term is derived, the latter acting as a small apt perturbation of the system dynamics. Implications of these findings are discussed in relation to the optimization of the laser source.Copyright (C)2006 IFAC
\end{abstract}

Keywords: laser, control

\section{INTRODUCTION}

A Free Electron Laser (FEL) generates a tunable, coherent, high power radiation. A FEL differs from conventional lasers in using a relativistic electron beam as its lasing medium. The physical mechanism responsible of the light emission and amplification is the interaction between a relativistic electron beam and a magnetostatic periodic field generated in the undulator. Due to the effect of the magnetic field, the electrons are forced to follow sinusoidal trajectories, thus emitting synchrotron radiation. This initial seed, termed spontaneous emission, is then amplified along the undulator until the laser effect is reached.

Among different schemes, Single-Pass, high-gain FELs are particularly attractive since they hold the promise of resulting in flexible light sources of broad theoretical and applied interests. The

\footnotetext{
1 Unité Mixte de Recherche (UMR 6207) du CNRS, et des universités Aix-Marseille I, Aix-Marseille II et du Sud Toulon-Var. Laboratoire affilié à la FRUMAM (FR 2291).
}

coupled evolution of the radiation field and the $N$ particle beam in a Single-Pass FEL can be successfully modeled within the framework of a simplified Hamiltonian picture Bonifacio, et al. (1990). The $N+1$ degree of freedom Hamiltonian displays a kinetic contribution, associated with the particles, and a potential term accounting for the self-consistent coupling between the particles and the field. Hence, direct inter-particles interactions are neglected, even though an effective coupling is indirectly provided because of the interaction with the wave.

The theory predicts a linear exponential instability and a late oscillating saturation, for the amplitude of the radiation field. Inspection of the asymptotic phase-space suggests that a bunch of particles gets trapped in the resonance and forms a clump that evolves as a single macro-particle localized in space. The remaining particles are almost uniformly distributed between two oscillating boundaries, and populate the so called chaotic sea. 
This observation allowed to derive a simplified Hamiltonian model to characterize the saturated evolution of the laser. Such reduced formulation consists in only four degrees of freedom, namely the wave, the macro-particle and the two boundaries, delimiting the portion of space occupied by the the uniform halo surrounding the inner bulk, see Antoniazzi et al. (2005) .

Furthermore, the macro-particle rotates around a well defined fixed point and this microscopic dynamics is shown to be responsible for the macroscopic oscillations observed at the intensity level. It can be therefore hypothesized that a significant reduction in the intensity fluctuations can be gained by implementing a dedicated control strategy, aimed at confining the macro-particle in space. In addition, the size of the macroparticle is directly related to the bunching parameter, a quantity of paramount importance in FEL context, see Antoniazzi and Fanelli (2005).

Smith and Pereira (1978) showed that a test-wave, being characterized by a frequency close to the one of the wave, can effectively destroy the macroparticle and activate a consequent detrapping process. Dimonte and Malmberg (1977) implemented this approach on a Travelling Wave Tube and detected a significant reduction of the intensity oscillations, followed, however, by an undisired systematic collapse of its mean-value.

In this paper, we focus on the macroparticle, spontaneosuly established as a result of the wave particle interaction process. In particular we develop a dedicated technique to influence and, possibly, control its evolution, thus opening up the perspective of defining innovative approaches aiming at stabilizing the laser signal. To this end, we consider a test-particle Hamiltonian in a mean-field approach and calculate a small, but appropriate, control term which acts as a perturbation. The latter is shown to induce an increase in size of the macro-particle. A regularization of the dynamics is also observed, as confirmed by the reconstruction of the invariant tori around the massive core.

The paper is organized as follows. In Sec. 1, we introduce the mean-field model and shortly outline its derivation from the original $N$-body Hamiltonian. In Sec. 2, a test-particle is "controlled", through the reconstruction of invariant tori of its dynamics. Finally, we draw our conclusion and discuss possible implication of the present analysis.

\section{THE MEAN-FIELD MODEL}

As previously anticipated, the dynamics of a Single-Pass FEL is successfully captured by the

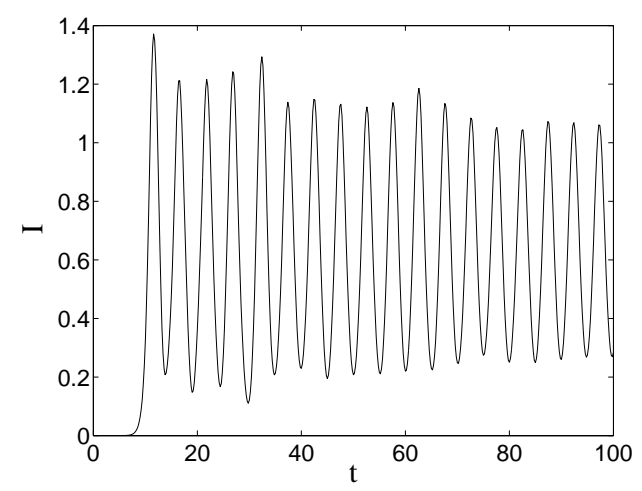

Figure 1. Normalized intensity of the FEL's radiation simulated from Hamiltonian (1).
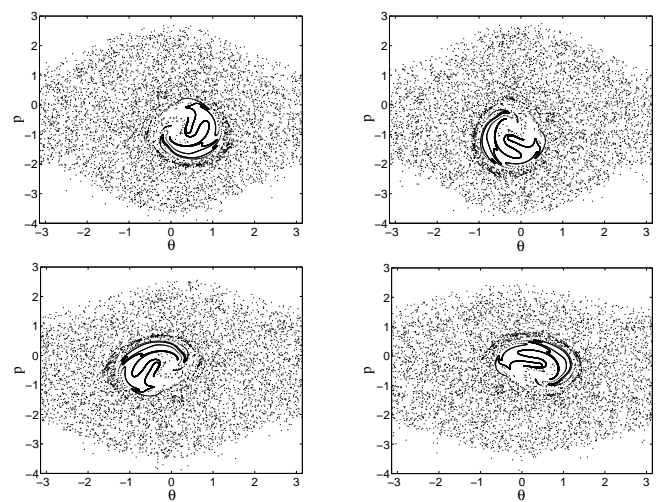

Figure 2. Phase space of the $N$ particles at $t=$ $400,401.25,402.5$ and 403.75 .

following Hamiltonian introduced by Bonifacio and collaborators (1990):

$$
H_{N}=\sum_{i=1}^{N}\left[\frac{p_{i}^{2}}{2}-2 \sqrt{\frac{I}{N}} \cos \left(\theta_{i}+\phi\right)\right],
$$

where $\left(\theta_{i}, p_{i}\right)$ are the position and momentum of the $i$-th particle and $(\phi, I)$ stand respectively for the phase and intensity of the radiation. In the mean-field model, the conjugated variables $(\phi, I)$ are replaced by two functions of time $\phi(t)$ and $I(t)$, obtained from direct simultations of the selfconsistent dynamics. In other words, this amounts to formally neglect the action of the electrons on the field.

The $N$-body Hamiltonian (1) can be therefore mapped into

$$
\tilde{H}_{N}=\sum_{i=1}^{N} H_{1 p}\left(\theta_{i}, p_{i}, t\right),
$$

where

$$
H_{1 p}(\theta, p, t)=\frac{p^{2}}{2}-2 \sqrt{\frac{I(t)}{N}} \cos (\theta+\phi(t)) .
$$

The dynamics of a FEL can be hence adressed by studying the evolution of a test particle, obeying to the Hamiltonian (2). $I(t)$ and $\phi(t)$ act as external parameters and are here imposed by assuming their simplified asymptotic behaviour as 
obtained from a frequency analysis (Laskar, 1999)

$$
2 \sqrt{\frac{I(t)}{N}} \approx F-\epsilon \sum_{k=1}^{K} W_{k} e^{i \omega_{k} t},
$$

in the referential of the wave.

\section{HAMILTONIAN CONTROL OF A TEST-PARTICLE}

The Hamiltonian control adresses to systems which are close to integrable, i.e. whose Hamiltonian can be written $H=H_{0}+V$, where $H_{0}$ is integrable and $V$ a perturbation of order $\epsilon$ (compared to $\left.H_{0}\right)$. The results we use here have been proven rigorously (Vittot, 2004; Vittot et al., 2005). In practice, it can be shown that a suitable control term $f$, of order $\epsilon^{2}$, exists such that $H_{0}+V+f$ has an invariant torus at a given frequency $\omega_{0}$. In our case, the perturbation corresponds to the oscillating part of the intensity. The interaction term of Hamiltonian (2) reads

$2 \sqrt{\frac{I(t)}{N}} \cos (\theta-\phi(t))=F \cos \theta-\epsilon R e\left(e^{i \theta} W(t)\right)$.

Thus, our integrable Hamiltonian can be cast in the form

$$
H_{0}=\frac{p^{2}}{2}-F \cos \theta
$$

whereas the time-dependent perturbation $V$ is identified as

$$
V(t, \theta)=\epsilon \operatorname{Re}\left(e^{i \theta} W(t)\right) .
$$

In its action-angle variables $(\varphi, J)$ (Lichtenberg and Libermann, 1983), Hamiltonian (3) can be expanded around $J=J_{0}$, which in turn identifies the region where the invariant torus is reconstructed in.

$H_{0}(J)=E_{0}+\omega_{0}\left(J-J_{0}\right)+\delta\left(J-J_{0}\right)^{2}+O\left(\left(J-J_{0}\right)^{3}\right)$.

Likewise, the $\theta$-component of perturbation (4) is expanded as

$$
e^{i \theta}=\sum_{m=0}^{M} \sum_{n=-L}^{L} \alpha_{m, n}\left(J-J_{0}\right)^{m} e^{i n \varphi}+\text { h.o.t. }
$$

The control term reads (Vittot et al., 2005) :

$f(\varphi, t)=V\left(J_{0}, \varphi, t\right)-V\left(J_{0}-\Gamma \partial_{\varphi} V\left(J_{0}, \varphi, t\right), \varphi, t\right)$, where $\Gamma$ is a linear operator acting on an element of the Fourier basis as :

$$
\Gamma e^{i(\omega t+n \varphi)}=\frac{e^{i(\omega t+n \varphi)}}{i(\omega+n)} .
$$

At the second order in $\epsilon$ (which is the main term), the control term reads

$$
f(\varphi, t)=\epsilon^{2} w(\varphi, t) \Gamma \partial_{\varphi} v(\varphi, t)-\epsilon^{2} \delta\left(\Gamma \partial_{\varphi} v(\varphi, t)\right)^{2},
$$
where

$$
\Gamma \partial_{\varphi} v(\varphi, t)=\sum_{k, n} \frac{n \alpha_{0, n} W_{k}}{\omega_{0} n+\omega_{k}} e^{i\left(n \varphi+\omega_{k} t\right)},
$$

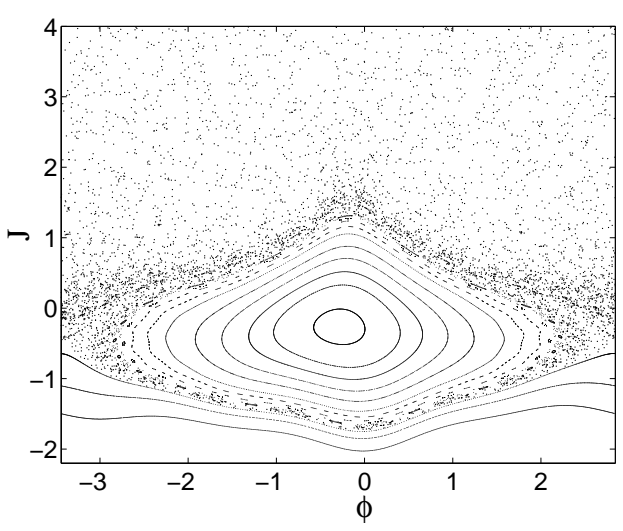

Figure 3. Poincaré section of the uncontrolled $\left(H_{0}+V\right)$ dynamics of a test particle, in action-angle variables.

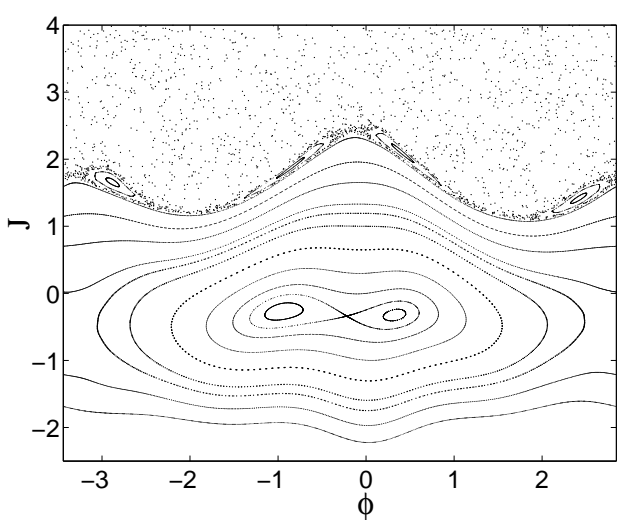

Figure 4. Poincaré section of the controlled $\left(H_{0}+\right.$ $V+f)$ dynamics of a test particle, in actionangle variables.

and

$$
w(\varphi, t)=\sum_{k, n} \alpha_{1, n} W_{k} e^{i\left(n \varphi+\omega_{k} t\right)} .
$$

Numerically, two Fourier modes were considered for $W(t)(K=2)$, and eleven for the $\theta$-dependent part of the perturbation (4) $(L=5)$. The parameter of the perturbation is of order $\epsilon=1 / 5$. The development is performed around $J_{0} \approx 1.33$ (which corresponds to energy $E_{0}=0$ ).

In action-angle variables, the regularization of the dynamics (see Figs.3 and 4) is manifest. Rather than a single torus, the control generated a continuous set of invariant tori, thus expanding the regular domain of the phase space.

Unfortunately, the exact change of variables from $(\varphi, J)$ to $(\theta, p)$ (see Lichtenberg and Libermann, 1983) has a singularity at the pendulum separatrices. In order to implement our control on the whole dynamics, we use a simplified, but regular, change of variables which mimics the exact one in the region of the invariant torus predicted by the control (the error in this region is less than 4\%) (see Fig.5) :

$$
\tan \varphi=\frac{\theta}{p}
$$




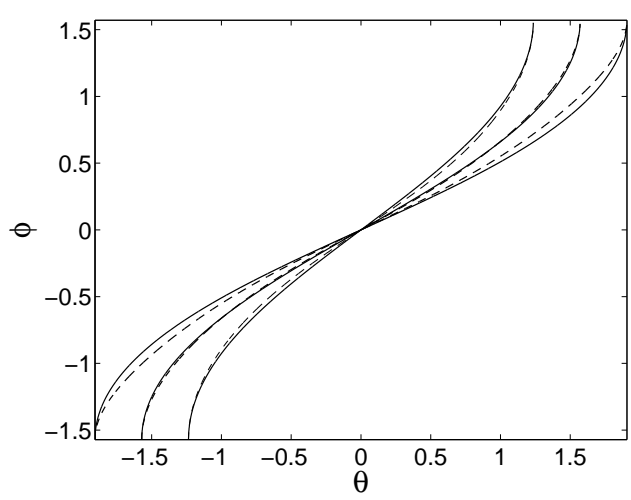

Figure 5. Plot of the exact and simplified changes of variables : $\varphi$ is plotted as a function of $\theta$, at constant energy, i.e. at constant $J$. The solid lines corresponds to the exact change of variables, the dashed ones to the simplified. The curves corresponds (from left to right) to $E=0.5,0$ and -0.5 .

In terms of the $(\theta, p)$ variables, our control term now reads :

$$
\tilde{f}(\theta, p, t)=f\left(\arctan \frac{\theta}{p}, t\right),
$$

and its regularity is now the one of the function $(\theta, p) \mapsto \arctan \frac{\theta}{p}$. Therefore, the controlled dynamics of a test-particle is given by Hamiltonian

$$
H_{c}(\theta, p, t)=H_{1 p}(\theta, p, t)+\tilde{f}(\theta, p, t)
$$

In the $(\theta, p)$ variables (see Figs. 6 and 7 ), the control is successful in reconstructing some invariant tori around the macro-particle. In other words, it enlarges this regular structure.

\section{CONCLUSION}

In this paper, we considered a simplified meanfield approach to investigate the saturated dynamics of a Single Pass FEL. In particular, we showed that the size of the macro-particle can be increased by adding a small pertubation to the system, thus resulting in a low cost correction in term of energy. The main idea is to build invariant tori localized in specific positions: this method is utterly general and could be succesfully used to adjust the size of the macro-particle, thus possibly enhancing the bunching factor. Analogously, by limiting the portion of phase-space spanned by the macro-particle one could aim at stabilizing the laser signal. Two future lines of investigation are foreseen. First, we intend to implement the computed control term in the framework of the original $N$-body self-consistent picture and explore possible beneficial effects for the evolution of the radiation field. Further, it is here planned to apply the above technique to the case of the reduced Hamiltonian of Antoniazzi and Fanelli (2005). We point out that an experimental test of

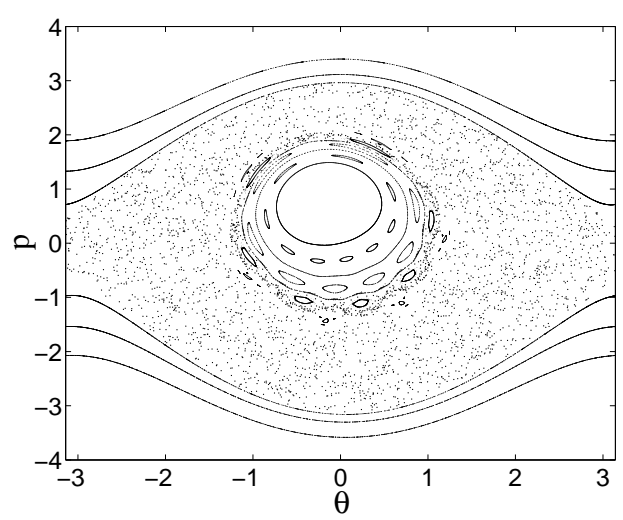

Figure 6. Poincaré section of the uncontrolled dynamics of a test particle, in the $(\theta, p)$ variables.

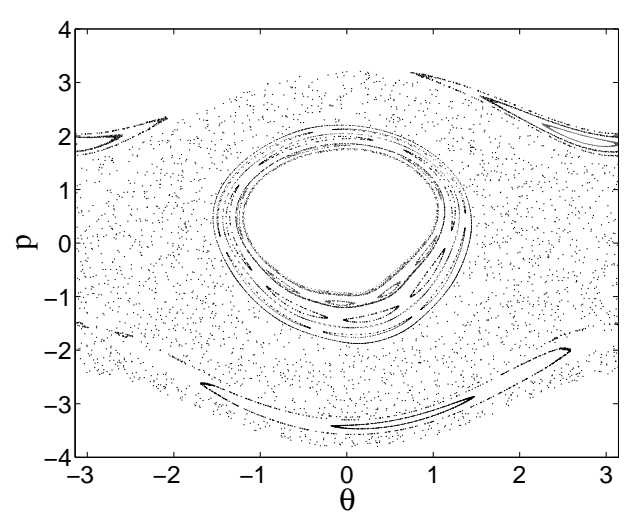

Figure 7. Poincaré section of the controlled dynamics of a test particle, in the $(\theta, p)$ variables.

the control method on a modified Travelling Wave Tube has been done by Chandre and collaborators (2005) in absence of self-consistency.

\section{REFERENCES}

Antoniazzi, A. and D. Fanelli (2005). Statistical mechanics and Vlasov equation allows for a simplified Hamiltonian description of Single-Pass Free Electron Laser saturated dynamics, submitted to J. Phys. A.

Bonifacio, R., et al. (1990). Physics of the highgain FEL and superradiance. Rivista del Nuovo Cimento, 3, pp. 1-69.

Chandre, C., G. Ciraolo, F. Doveil, R. Lima, A. Macor and M. Vittot (2005). Channeling chaos by building barriers. Phys. Rev. Lett., 74, 074101.

Dimonte, G., J.H. Malmberg (1977). Destruction of Trapped-Particle Ocillations. Phys. Rev. Lett., 38, pp. 401-404.

Laskar, J. (1999). Introduction to Frequency Map Analysis. Proc. of NATO ASI Hamiltonian Systems with Three or More Degrees of Freedom, (C. Simò Ed, Kluwer), pp. 134-150. 
Lichtenberg, A.J. and M.A. Libermann (1983). Regular and Stochastic Motion. Springer, New York.

Smith, G.R. and N.R. Pereira (1978). Phaselocked particle motion in a large-amplitude plasma wave. Phys. Fluids, 21, pp. 2253-2262.

Tennyson, J.L., J.D. Meiss, and P.J. Morrison (1994). Self-Consistent Chaos in the BeamPlasma Instability. Physica D, 71, pp. 1-17.

Vittot, M. (2004). Perturbation theory and control in classical or quantum mechanics by an inversion formula. J. Phys. A: Math. Gen., 37, pp. 6337-6357.

Vittot, M., C. Chandre, G. Ciraolo and R. Lima (2005). Localized control for non-resonant Hamiltonian systems. Nonlinearity, 18, pp. 423-440. 